\title{
Towards a more accurate quantification of human-environment interactions in the past
}

\author{
Gert Verstraeten \\ Leuven, Belgium, 3-7 February 2014
}

\begin{abstract}
Since the introduction of agriculture, humans have profoundly changed the natural environment through land cover change and subsequent changes in soil and sediment properties as well as aquatic ecosystems. It is also clear now that these long-term changes are also responsible for significant changes in the global carbon cycle with feedbacks to climate. The PAGES Themes on Soil and Sediment (formerly LUCIFS) and Land Use and Cover have made great progress in the last decade in reconstructing sediment fluxes under human impact and anthropogenic land cover changes, respectively. However, despite the progress these groups have made, we still have a very incomplete picture of human-environment interactions over the Holocene, both spatially and temporally. There is a need for further integration of the various disciplines active under Focus 4 and inclusion of scientists from the humanities.
\end{abstract}

This workshop provided an interdisciplinary forum to share data, results and ideas in order to explore how our research community should proceed to work towards an integrated quantitative history of Holocene human-environment interactions. In total 105 researchers from 19 countries and from a wide variety of disciplines such as geography, archeology, palynology, history, geology and soil sciences contributed to the workshop.

The first two days of the workshop were dedicated to 31 oral and 57 poster presentations, which highlighted new research results on specific topics of past human-environment interactions to a wider disciplinary audience. These sessions included 10 invited speeches. Topics included the quantification and numerical modeling of past changes in vegetation cover, population and settlement dynamics, changes in agricultural techniques and the use of innovative geochemical and isotopic techniques for tracing historic soil erosion and sedimentation.

On the third day of the workshop, Jean Poesen, Gert Verstraeten, Bastiaan Notebaert and Nils Broothaerts (Leuven, Belgium) led a field trip through the Dijle River catchment in the Belgian Loess Belt to display and discuss how humans have changed the landscape through deforestation and subsequent erosion and sedimentation processes (Fig. 1)

The last two days of the workshop were entirely devoted to discussions. A first series of discussion sessions were organized in breakout groups and restricted to the disciplinary topics "archeology", "land cover", "geomorphology" and "paleolimnology". These groups not only discussed the state-of-the-art of each of these broad disciplines with respect to their understanding of human-environment interactions but also how each discipline could contribute to the overall questions on human-environment interactions. They also identified the major knowledge gaps and formulated some key questions for further research. Each group presented the outcome of the discussion to the entire workshop audience.

In a second step, three topical, cross-disciplinary discussion sessions, again in breakout groups were organized. One group focused on scale issues and the uncertainties involved when reconstructing past human-environment interactions, whilst another group discussed the role of thresholds or tipping points in complex human-environment systems and the feedback mechanisms that come into play. A third group discussed the difficulties that arise to define human impact through time and space considering important changes and variability in agricultural techniques, productivity, economy and culture.

During the final plenary session that concluded the workshop, the participants discussed future research strategies and synergies that fit within the Future Earth research initiative (www. futureearth.org). The participants stressed the importance of knowing more about past human-environmental interactions for future land planning and sustainable management of natural resources, and the need for environmental and humanities-based disciplines to join forces and move beyond overly-simplistic and uni-directional views on how humans have impacted the environment and vice versa. The interdisciplinary audience also endorsed moving towards a more quantitative perspective on past human-environment interactions in order to improve forecasts of future human-environment interactions.

\section{PROGRAM AND ABSTRACTS}

www.pages-igbp.org/

calendar/2014/127-pages/827-focus-4-wshop

\section{AFFILIATIONS}

Department of Earth and Environmental Sciences \& Center for Archaeological Sciences, University of Leuven, Belgium

\section{CONTACT}

Gert Verstraeten: gert.verstraeten@ees.kuleuven.be

REFERENCES

Verstraeten G (2012) Neth J Geosci 91: 233-244
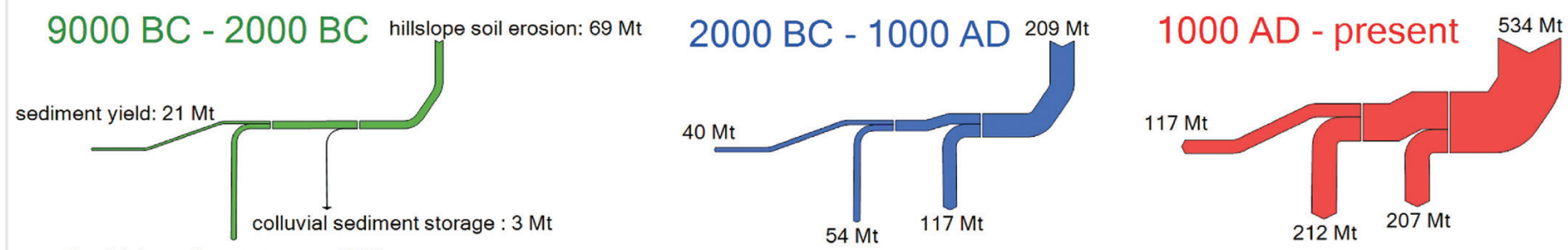

floodplain sediment storage: $45 \mathrm{Mt}$

Figure 1: One of the themes discussed during the workshop is the quantification of historic soil erosion and sedimentation following human disturbance. The timedifferentiated sediment budget for the Dijle catchment shows the changing importance of colluvial and alluvial storage as well as sediment yield through time. Not only the absolute increase in human-induced land cover change, but also its spatial patterns explain the observed changes in the sediment record. The entire system behaves nonlinearly and is characterized by tipping points, which change the coupling relationship between hillslopes, river channels and floodplains. Adapted from Verstraeten (2012). 